\title{
3D CONFOCAL MICROSCOPY DATA ANALYSIS USING LEVEL-SET SEGMENTATION WITH ALPHA DIVERGENCE SIMILARITY MEASURE
}

\author{
Leila Meziou $^{1}$, Aymeric Histace ${ }^{1}$, Frédéric Precioso ${ }^{2}$, Bogdan J. Matuszewski ${ }^{3}$ and Franck Carreiras ${ }^{4}$ \\ ${ }^{1}$ ETIS UMR 8051 CNRS /ENSEA/ Cergy-Pontoise University, 95000 Cergy, France \\ ${ }^{2}$ I3S - UMR 6070 - CNRS/Nice Sophia Antipolis University, France \\ ${ }^{3}$ ADSIP Research Centre, University of Central Lancashire, Preston, UK \\ ${ }^{4}$ ERRMECe, Cergy-Pontoise University, 95000 Cergy, France \\ \{leila.meziou,aymeric.histace\}@ensea.fr, precioso@polytech.unice.fr, bmatuszewski1@uclan.ac.uk, \\ franck.carreiras@u-cergy.fr
}

\begin{abstract}
Keywords: Image Segmentation : Active Contours : Alpha-Divergence : Level-Set : Confocal Microscopy.
Abstract: $\quad$ Segmentation of cellular structures is of primary interest in cell imaging for a 3D reconstruction of cell shape. Such an analysis provides crucial information about cell morphology and is instrumental in understanding of biological processes leading to development of a particular pathology. The work presented in this paper reports on a novel method for segmentation of cellular structures (nuclei and cell boundaries) from 3D single channel actin tagged fluorescence confocal microscopy images. The proposed segmentation method uses histogram-based image similarity measure in a level-set active-contour framework. The novelty of the method is in application of the alpha-divergence distance measure which can be seen as a generalization of classic Kullback-Leibler and $\chi^{2}$ measures. The resulting alpha-divergence level-set formulation leads to a single front evolution formula for both nuclei and cell boundaries segmentation, with no requirements for any enhancement or preprocessing of acquired cell images (a monolayer of human cells (PNT2) culture).
\end{abstract}

\section{INTRODUCTION}

Segmentation of cellular structures is an essential tool in cell imaging as it enables measurements which can be used to track cell divisions or help to reconstruct corresponding cell lineage tree providing data for calculation of different parameters like cell proliferation rate for instance. More specifically, the work presented in this paper has been carried out in a context of analyzing changes of cell cytoskeleton properties in a response to ionizing radiation insult. The final goal of this research effort is to better understand cell bio-mechanical responses during cancer radiation therapy. Indeed, actin tagged fluorescence confocal microscopy imaging enables to characterize important properties of cytoskeleton, in particular actin filaments which are involved in many cellular processes like cell adhesion, locomotion, inter-cell transport and general cell structural integrity, to name a few. Nevertheless, due to a highly complex actin appearance, a high level of noise and a strong nonhomogeneity of intensity and gradient information, the segmentation of cell structures in such imaging data, is a very challenging task. In this context, we propose a (quasi-)automatic segmentation approach, reducing to a minimum manual interventions - which represent practical bottleneck when considering many monolayer acquisition - in order to extract nuclei and cell boundaries that provide spatial reference frame for analyzing cytoskeleton changes.

To date, only few methods propose to address segmentation of cell structures in fluorescence confocal microscopy images (FCMI). In former approach proposed in (Ortiz De Solorzano et al., 1999), authors focused on nuclei segmentations. In (Yan et al., 2008) authors proposed cell segmentation in 2D-fluorescent images with two channels (actin and nucleus tagging) using a multiphase level-set combining ChanVese (Chan and Vese, 2001) and geodesic active contour models, together with repulsive force introduced to prevent segmented cells from overlapping. In (Mosaliganti et al., 2009; Zanella et al., 2010), automated 3D cell segmentation from 3D confocal acquisition of early Zebrafish embriogenesis is proposed, two different fluorescent markers (red for nu- 
clei and green for membrane) are used to easily discriminate nuclei from cell membranes. In (Zanella et al., 2010), authors introduced an adapted version of the subjective surface technique (Sarti et al., 2002) for surface reconstruction from missing boundary information whereas (Mosaliganti et al., 2009) use a multiphase level-set based on propability correlation functions.

Although our method is somewhat similar to (Yan et al., 2008; Mosaliganti et al., 2009) focusing on level-set framework, the objective is different since our microscopic 2D images are extracted from a 3D single channel confocal acquisition with only one fluorescent marker used for actin tagging making it a more challenging problem. Nuclei and cell boundaries segmentations are considered as two separate tasks but both use the same Partial Differential Equation (PDE) describing evolution of the active contour. Finally, as it will be shown, no enhancement or preprocessing will be required to segment acquired images.

The data used in this paper were obtained from human prostate cells (PNT2). Once confluent cells were fixed, actin were labelled with phalloidin-FITC according to the manufacturers instructions (Invitrogen, UK). All imaging was carried out using a Zeiss LSM510 confocal microscope. Fig. 1 shows some images extracted at different slice levels from the 3D microconfocal acquisition of the monolayer PNT2 cell culture. The stack volume is defined on the $512 \times 512 \times 98$ grid of pixels each $0.21 \mu \mathrm{m} \times 0.21 \mu \mathrm{m}$ $\times 0.11 \mu$ in size.

The remaining of this paper is organized as follows: in Section 2, the alpha-divergence measure and our histogram-based active contour segmentation approach are explained together with the derivation of the corresponding governing PDE in the level-set framework; Section 3 focuses on experiments followed by conclusions drawn in Section 4.

\section{THEORETICAL FRAMEWORK}

Originally proposed in (Kass et al., 1988), the basic idea of the active contour is to iteratively evolve an initial curve towards the boundaries of target objects driven by the combination of internal forces, determined by the geometry of the evolving curve, and external forces, induced from the image. Image segmentation methods using active contour are often derived from a variational principle in which a functional defined on contours encodes our knowledge about desirable solution. The functional minimization leads to a partial differential equation (PDE), constructed as the Gateaux derivative gradient flow which steers the evolution of the active contour.
In the particular framework of a region-based active contour segmentation (Aubert et al., 2003), several functional definitions have been proposed to take into account the Probability Density Functions (PDFs) of both the inner and outer regions of the evolving curve. The corresponding variational criterion is based on the minimization of a distance between PDFs calculated for the inner and outer regions as defined by the evolving contour and predefined reference PDFs of targeted and background objects. Common distances used to compare PDFs are, for instance, the $\chi^{2}$ distance (Aubert et al., 2003) or the Kullback-Leibler divergence (Lecellier et al., 2010). In this paper, we propose an original histogram-based active contour approach integrating alpha-divergence as distance to minimize between two PDFs which can be estimated parametrically or not. Let $\hat{q}$ denotes an estimated PDF and $p$ a reference one for a particular region $\Omega$ extracted from the image. As $\hat{q}$ represent the current PDF, it is non-parametrically estimated using Parzen window method. This choice is motivated by the fact that $\hat{q}$ has to be differentiable for the next calculation step of the minimization scheme. Distance between $\hat{q}$ and $p$ using the alpha-divergences is now defined as follows (Beirami et al., 2008):

$$
D_{\alpha}(\hat{q} \| p, \Omega)=\int_{\mathfrak{R}^{n}} \varphi_{\alpha}(\hat{q}(\lambda, \Omega), p(\lambda)) d \lambda,
$$

with $\varphi_{\alpha}$ the cost function related to alpha-divergence measure defined by:

$$
\begin{aligned}
\varphi_{\alpha}(\hat{q}(\lambda, \Omega), p(\lambda)) & =\frac{1}{\alpha(1-\alpha)}(\alpha \hat{q}(\lambda, \Omega)+(1-\alpha) p(\lambda) \\
& \left.-[\hat{q}(\lambda, \Omega)]^{\alpha}[p(\lambda)]^{1-\alpha}\right)
\end{aligned}
$$

where $\alpha \in \Re$.

A complete study about the mathematical properties of alpha-divergence can be found in (Beirami et al., 2008). Nevertheless, considering Eq. (2), for specific values of $\alpha$, some aforementioned standard distances can be connected to alpha-divergences, for instance: $D_{2}(\Omega)=\frac{1}{2} D_{\chi^{2}}(\Omega), D_{\frac{1}{2}}(\Omega)=2 D_{\text {Hellinger }}(\Omega)$, $D_{K L}(\Omega)=\frac{1}{2}\left(\lim _{\alpha \rightarrow 0} D_{\alpha}(\Omega)+\lim _{\alpha \rightarrow 1} D_{\alpha}(\Omega)\right)$.

In the general framework of the histogram-based active contours the alpha-divergence functional can now be defined as follows for grayscale images $(n=$ 1):

$$
\begin{aligned}
J_{\alpha}\left(\Gamma, \Omega_{\text {in }}, \Omega_{\text {out }}\right) & =\xi_{\text {in }} \int_{\Re} \varphi_{\alpha}\left(\hat{q}\left(\lambda, \Omega_{\text {in }}\right), p_{\text {in }}(\lambda)\right) d \lambda \\
& +\xi_{\text {out }} \int_{\Re} \varphi_{\alpha}\left(\hat{q}\left(\lambda, \Omega_{\text {out }}\right), p_{\text {out }}(\lambda)\right) d \lambda \\
& +\beta \int_{\Gamma} d s
\end{aligned}
$$

where $\Omega_{\text {in }}$ and $\Omega_{\text {out }}$ are respectively the foreground (targeted object) and the background areas, $\Gamma$ the 
evolving boundary between $\Omega_{\text {in }}$ and $\Omega_{\text {out }}$ and $\xi_{\text {in }}$, $\xi_{\text {out }}$ and $\beta$ three positive weighting parameters. Considering now the standard level-set embedding function $U$ of $J_{\alpha}$, the Lagrangian minimization scheme of $J_{\alpha}$ leads to the following associated PDE for active contour evolution:

$$
\begin{aligned}
\frac{\partial U}{\partial t}=\delta U\left(\beta \nabla \cdot\left(\frac{\nabla U}{|\nabla U|}\right)\right. & -\frac{\xi_{\text {in }}}{\left|\Omega_{\text {in }}\right|}\left(A_{\text {in }}-C_{\text {in }}\right) \\
& \left.+\frac{\xi_{\text {out }}}{\left|\Omega_{\text {out }}\right|}\left(A_{\text {out }}-C_{\text {out }}\right)\right)
\end{aligned}
$$

with

$$
\begin{aligned}
& A_{i}=\partial_{1} \varphi_{\alpha}\left(\hat{q}\left(\lambda, \Omega_{i}\right), p_{i}(\lambda)\right) * g_{\sigma}(\mathbf{I}(\mathbf{x})), \\
& C_{i}=\int_{\Re} \partial_{1} \varphi_{\alpha}\left(\hat{q}\left(\lambda, \Omega_{i}\right), p_{i}(\lambda)\right) \hat{q}\left(\lambda, \Omega_{i}\right) d \lambda,
\end{aligned}
$$

where $i=\{$ in, out $\}$ and $\partial_{1} \varphi_{\alpha}$ denotes the first derivative order of $\varphi_{\alpha}$ function with respect to $\hat{q}, g_{\sigma}$ is the Gaussian kernel (with standard-deviation $\sigma$ ) used in the Parzen window estimation of $\hat{q}, \mathbf{I}$ is the intensity function of the segmented image at a pixel $\mathbf{x}$. The implementation of Eq. (4) is achieved with a semi-implicit version of the Additive Operator Splitting scheme.

\section{EXPERIMENTAL RESULTS}

In order to achieve the automatic segmentation of both nuclei and cell boundaries, inner and outer reference PDFs, corresponding to the targeted structures, are computed applying a standard three class (for cell boundaries, cytoplasm and nucleus) ExpectationMaximization (EM) algorithm on the equator slice of the PNT2 acquisition. This rough classification is good enough to define the reference PDFs using Parzen window technique: the strategy is then one class ("nucleus", resp. "cell boundaries") against the two others ("cell boundaries+cytoplasm", resp."nucleus+cytoplasm"). The segmentation process is initialized on the equator slice (Fig. 1 mid column) through the monolayer. For both nuclei and cell boundaries segmentations, the zero level-set initialization of the function $U_{0}$ is given as a set of small circles uniformly distributed across the whole slice. Finally, the segmentation process is spread all along the different slice level of the monolayer.

Fig. 1 summarizes results obtained for different slices from the acquired 3D image stack and for different values of the parameter $\alpha$. All tests are done with the same optimal parameters $\xi_{i}$ (here $\xi_{\text {in }}=\xi_{\text {out }}$ ) and $\beta$ for both nuclei and cell boundaries (empirically tuned on the equator slice image) in order to focus on analysis the influence of the $\alpha$ parameter only.

Considering nuclei segmentation, as shown in Fig. 1 in green, thanks to the level-set formulation and to the integration of alpha-divergence measures, all nuclei are detected during a single run of the algorithm, even those with incomplete shape (at the border of image). This is a major advantage when comparing with the Chan-Vese approach which needs to be carefully adapted (in its regularized form) to perform the same task as shown in some of our previous work (Meziou et al., 2011) on that topic. It is also important to notice that the more accurate results are obtained with non-standard values of $\alpha$ as shown in Figs. 1. (b). where $\alpha=0.75$. The real difficulty is to obtain a robust segmentation result of each nucleus to avoid small structures segmentation within cytoplasm reflecting the complexity of the cytoplasmnucleus boundary. For the higher values, false detections could appear and non significant small structures are segmented as shown in Figs. 1. (e).

For each slice segmentation, an expert can manually suppress false detected nuclei that can occur when a hole representing an empty space between cells is present in the cell culture for instance: Statistically speaking, the PDF of a hole is very close to the PDF of a nucleus and can be difficult to differentiate automatically.

In the case of cell boundaries, shown in red in Fig. 1, segmentation is more demanding since even for experts it is not always easy to visually identified them: the non-homogeneity of the actin fluorescent marker can strongly influence the pixel levels corresponding to cell boundaries which could explain difficulties to get continuous contours. Results obtained are similar to those obtained with Chan and Vese segmentation which performed well for this particular task and can be considered as a reference for that kind of single channel acquisition. However, major advantage of the proposed approach for cell boundaries segmentation is the use of the same evolution criterion (alpha-divergence measure) for both cell boundaries and nucleus segmentations. Influence of the $\alpha$ parameter, as for nuclei segmentation, is interesting since it can be noticed again that the most interesting results are obtained for a value of $\alpha$ that does not correspond to standard histogram distance measures. In terms of cell boundaries segmentation, the proposed results have to be considered as prospective ones but very encouraging. From an expert point of view, the best results are obtained for a high value of $\alpha$ (for example $\alpha=1.5$ shown in Figs. 1.(d)).

\section{CONCLUSION}

In this paper a novel histogram based level-set active contours method is proposed for segmentation of nuclei and cell boundaries in 2D images from a 3D single channel confocal microscopic acquisition 

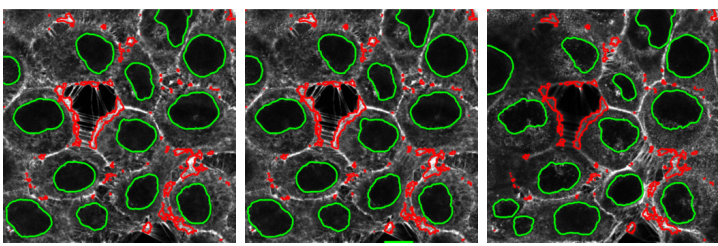

(a) $\alpha=0.5$.
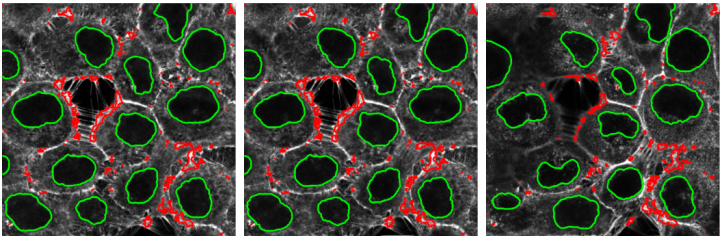

(b) $\alpha=0.75$
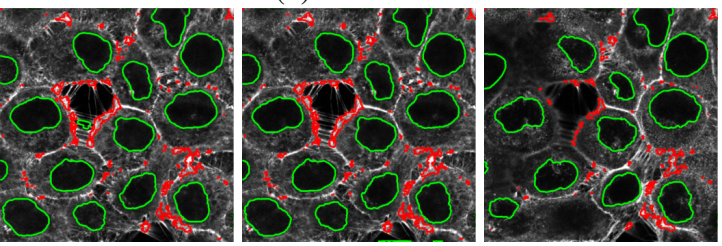

(c) Kullback-Leibler divergence
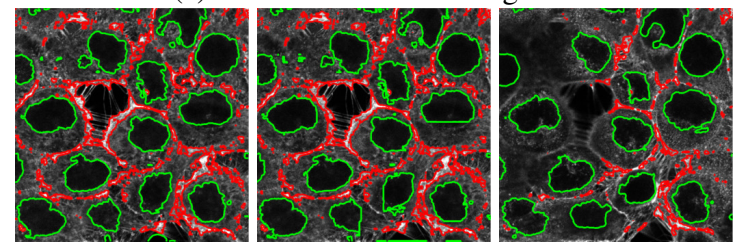

(d) $\alpha=1.5$.
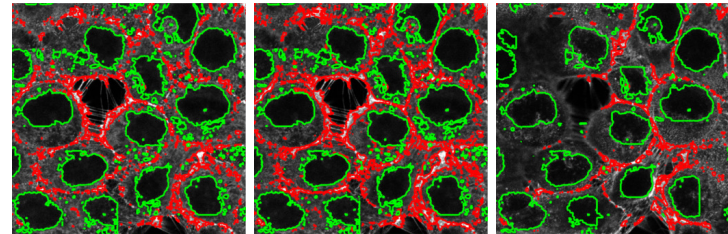

(e) $\alpha=2$

Figure 1: Segmentation of cell nuclei (in green) and cell boundaries (in red) with different values of $\alpha$ (row) and for different slice index in the acquired 3D image stack (column, from left to right slices from bottom, middle, and top of the stack).

of a PNT2 cells monolayer. Introduction of alphadivergence measure within the variational framework leads to a single evolution PDE for segmentation of both nuclei and cell boundaries. The segmentation results obtained for the nuclei and cell boundaries, show that the method enables to control in an efficient way a range of distances that can be used in very different segmentation scenarios. Moreover, compared to previous works, the proposed method does not require any enhancement or preprocessing since the performance of alpha-divergence measure can be adapted to the level of the corrupting noise and if expert manual interactions are permitted, they can be reduced to a minimum. Additionally, as already mentioned, the cell structure segmentation could be achieved in the particular case where only actin is tagged in opposition to other recent studies reported in the literature. It is envisaged that the future work will include: (i) improvement of cell boundaries segmentation using a local adaptation of $\alpha$ parameter based on noise characteristics of each classes, and (ii) development of a joint nuclei and cell boundaries 3D segmentation.

\section{ACKNOWLEDGEMENTS}

This work was supported by the UK Engineering and Physical Sciences Research Council [TeRaFS project, grant number EP/H024913/1].

\section{REFERENCES}

Aubert, G., Barlaud, M., Faugeras, O., and Jehan-Besson, S. (2003). Image segmentation using active contours: Calculus of variations or shape gradients? SIAM J. Appl. Math., 63:2128-2154.

Beirami, A., Cevher, V., Bower, B., and Tsianos, K. (2008). Proofs of alpha divergence properties. Technical Report STAT 631 / ELEC 639, Rice University.

Chan, T. F. and Vese, L. A. (2001). Active contours without edges. IEEE trans. on IP, 10(2):266-277.

Kass, M., Witkin, A., and Terzopoulos, D. (1988). Snakes: Active contour models. Int. J. Comput. Vision, V1(4):321-331.

Lecellier, F., Fadili, M., Jehan-Besson, S., Aubert, G., Revenu, M., and Saloux, E. (2010). Region-based active contours with exponential family observations. $J$. of Math. Imaging and Vision, 36(1):28-45.

Meziou, L., Histace, A., Precioso, F., Matuszewski, B., and Murphy, M. (2011). Confocal Microscopy Segmentation Using Active Contour Based on AlphaDivergence. In Proceedings of ICIP 2011, pages 3138-3141.

Mosaliganti, K., Gelas, A., Gouaillard, A., Noche, R., Obholzer, N., and Megason, S. (2009). Detection of spatially correlated objects in $3 \mathrm{~d}$ images using appearance models and coupled active contours. In Proceedings of MICCAI'09, pages 641-648, Berlin, Heidelberg. Springer-Verlag.

Ortiz De Solorzano, C., Garcia Rodriguez, E., Jones, A., Pinkel, D., Gray, J. W., Sudar, D., and Lockett, S. J. (1999). Segmentation of confocal microscope images of cell nuclei in thick tissue sections. Journal of Microscopy, 193(3):212-226.

Sarti, A., Malladi, R., and Sethian, J. A. (2002). Subjective surfaces: A geometric model for boundary completion. Int. J. Comput. Vision, 46(3):201-221.

Yan, P., Zhou, X., Shah, M., and Wong, S. T. C. (2008). Automatic segmentation of high throughput rnai fluorescent cellular images. IEEE Transactions on Information Technology in Biomedicine, 12(1):109-117.

Zanella, C., Campana, M., Rizzi, B., Melani, C., Sanguinetti, G., Bourgine, P., Mikula, K., Peyriéras, N., and Sarti, A. (2010). Cells segmentation from $3 d$ confocal images of early zebrafish embryogenesis. IEEE trans. on IP, 19(3):770-781. 